\title{
Applying Built-in Virtual Personal Assistant for Educational Equipment
}

\author{
Jinghui Yang and Yavor Stefanov \\ Department of Industry Engineering \\ Shanghai Polytechnic University \\ Shanghai, China \\ Jinghui.yang@sspu.edu.cn
}

\author{
Zhe Li and Kesheng Wang \\ Department of Production and Quality Engineering \\ Norwegian University of Science and Technology \\ Trondheim, Norway \\ \{zhe.li \& kesheng.wang\}@ntnu.no
}

\begin{abstract}
The report exposes an idea to solve a problem of different hardware development board with various software modules that enter more actively in the educational process. This paper focuses on the establishment of an educational device, which is compatible with multiple sensors, modules and interactive interfaces to merge different software platforms. A hardware module with built-in virtual personal assistant to help teach in the training of the digital generation will be presented. In addition, the device can also be compatible with platforms such as SAP HANA for additional system capabilities.
\end{abstract}

Keywords - hardware equipment; virtual personal assistant; the education system; digital generation

\section{INTRODUCTION}

The new generation has the opportunity today to choose different platforms to develop hardware devices. Students are inspiration to create new generation device based on endless development opportunities with the open source software and open hardware platforms. One of the challenges for the education system is to ensure better training of a new generation of available to them interactive form. Many organizations besides offering of hardware and software they also offer own platform of education. Some of them are:

Picademy is the Raspberry Pi Foundation's FREE teacher training initiative. Our goal is to give educators the experience and tools they need to teach computing with confidence [1]. Raspberry Pi Foundation has seven developer boards for different user applications.

Banana Pi targets to be a cheap, small and flexible enough computer for daily life. Built with ARM Cortex-A7 Dual-core CPU and Mali400MP2 GPU, and open source software, Banana Pi can serve as a platform to make lots of applications for different purposes [2]. Banana $\mathrm{Pi}$ organization have nine developer boards for different user applications.

The Beagle family is happiest when helping students to learn programming, hobbyists to push the boundaries of DIY and developers move to production quickly without excess noise, expense or bulk [3]. BeagleBone has available four developer boards.

Arduino and Genuino Education is a worldwide-leading school initiative bringing technology into the hands of teachers and students to create a more inventive learning experience [4]. The community of Arduino has more than twenty-five type of board in worldwide.

Designed for expert makers, entrepreneurs, and some industrial IoT applications, the Intel ${ }^{\circledR}$ Edison compute module provides ease-of-development for a range of prototyping projects or commercial ventures when performance matters [5]. Intel Corporation presents three model boards for developers.

These powerful platforms for development in combination with various sensors and modules are excellent for solving various problems. But these platforms have their disadvantages. When analyzing the current market there are many available sensors and modules built, but not compatible designs and interface. Also there are many platforms to develop hardware devices, but not with efficient use of information about working with them. Therefore the creating a device compatible with multiple sensors and modules and interactive interface will be in favor of the training. The device must have a suitable interactive interface for communication with users. This device also must be compatible with current standards in production lines like SAP - HANA. One of the main tasks is the creation of unified approach to training in different types of boards and software for developers.

The remaining part of the paper is organized as follows. Section 2 describes the hardware equipment of the device developed in this paper. The main components include development board, Human machine interface module, and power supply module. Section 3 discusses the built-in Virtual Personal Assistant (VPA), which unites the various software platforms and presents an interactive training model. Section 4 describes the integration and compatibility with application platforms for data analysis and processing under the environment of big data. Conclusions and future work are summarized in the last section of this paper.

\section{HARDWARE EQUIPMENT}

The education system needs a flexible platform for working with the incoming new technologies also interactive modern approach to training a new generation. Hardware equipment presented to the current article aims to focus on training using current popular technologies, but also promotes the use of new interactive approaches to learning. The presented model solves 
the problem of differences in hardware devices by creating a module that integrates relevant board, and presented in an accessible way I/O interface. The equipment is shown in Fig. 1. The software differences are eliminated by using the interactive approach of the virtual personal assistant. Using this new approach can focus attention on the experiment and upgrade their knowledge during the learning process.

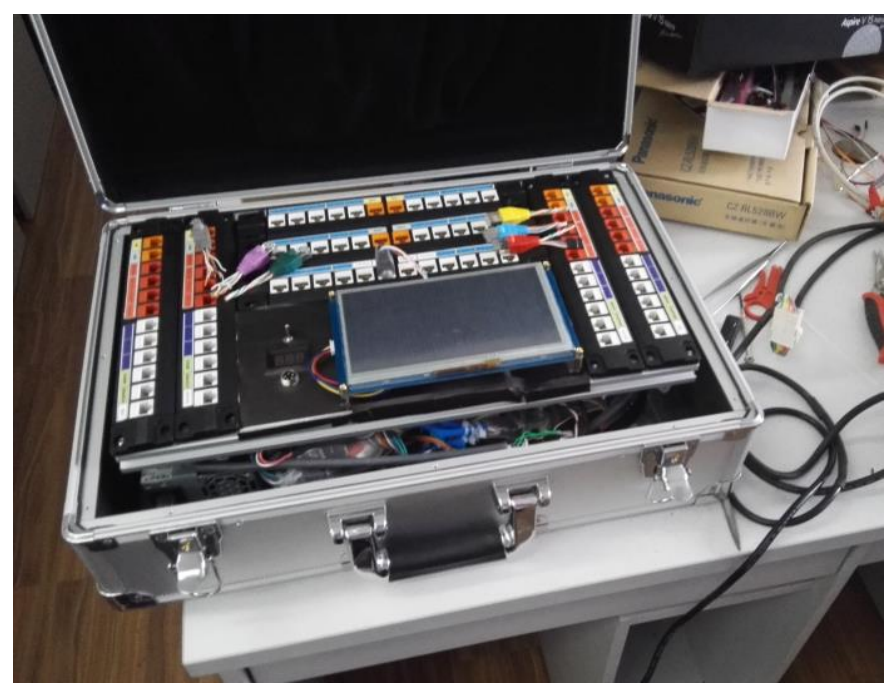

Fig. 1 Educational equipment with built-in Virtual Personal Assistant

Hardware conceptual model identifies key aspects of the work of the individual hardware components as shown in Fig. 2. The main hardware component is the "MAIN HARDWARE MODULE" which consists of the following components.

The main component of this core module is the development board, which may be a homogeneous system of several types of boards. The solution in this concert is the connection of different types of interfaces in the respective colors UF (Universal female) connectors. The different color of UF connectors are connected to different types of interfaces SPI, TWI, Digital, Analog, Network, Serial, etc.

The board for the development is linked HMI (Human machine interface) module via a serial interface. HMI module is main part to creating an interactive controls system for training the new generation.

All modules and UF are powered by power supply module, which includes the appropriate necessary protections.

One of the main ideas of this concept are also peripheral I/O devices which can be very different modules and sensors, by using the UM (Universal male) connector, respectively in a different color to use interface. One of the innovative ideas of the concept is the use of UF and UM connectors in different color depend of interface. This approach facilitates how to connect the main unit with many different modules and sensors without unnecessary connecting cables.
By using universal connectors UF and UM make it easy for the process of development and training as teachers and students are concentrated entirely on the respective development. Developed by this method models can easily build and upgrade can also be used repeatedly in different development or training modules. The available modules and sensors on the market could easily connect with UM connector and thereby hundreds of I/O devices can be used in development. This is the concept of open hardware and software, where everyone can develop a new peripheral device and offer different solutions to actual problems. One of the innovative ideas of this concert is the ability to combine several different platforms for developers in one or a combination of core modules with different platforms. It is precisely thus will be able to maximize the benefits of the different systems.

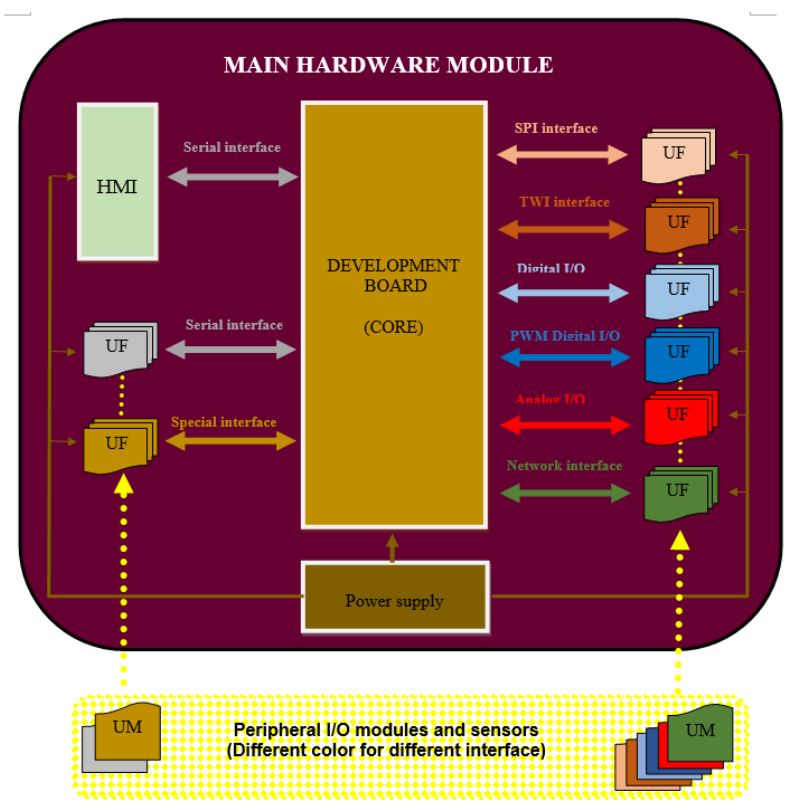

Fig. 2 Concept of hardware main module with peripheral I/O sensors

\section{BUILT-IN VIRTUAL PERSONAL ASSISTANT}

One of the challenges facing the education system creating a common model for merging different software platforms. Virtual Personal Assistant unite the various software platforms and presents an interactive training model. Digital generation shows better results when detects and makes by himself than to listen and watch passively the tasks. Built virtual personal assistant also helps to friendly directs and guides students for the upcoming tasks in this way they have the opportunity to show to his knowledge. The use of VPA in the educational process is viewed by the authors as an interactive application to help student's daily life using their smart devices $[6,7]$.

The first stage of the algorithm is to extract information from the student database. In the preset ID of the student extract data for specialty, training a group, running exercises, outcomes from previous exercises and more. These data are needed to VPA configure hardware and software equipment to the 
necessary requirements for exercise of any student. In this way the system is flexible and can be used in many different disciplines and students. The flowchart of the algorithm is shown in Fig. 3.

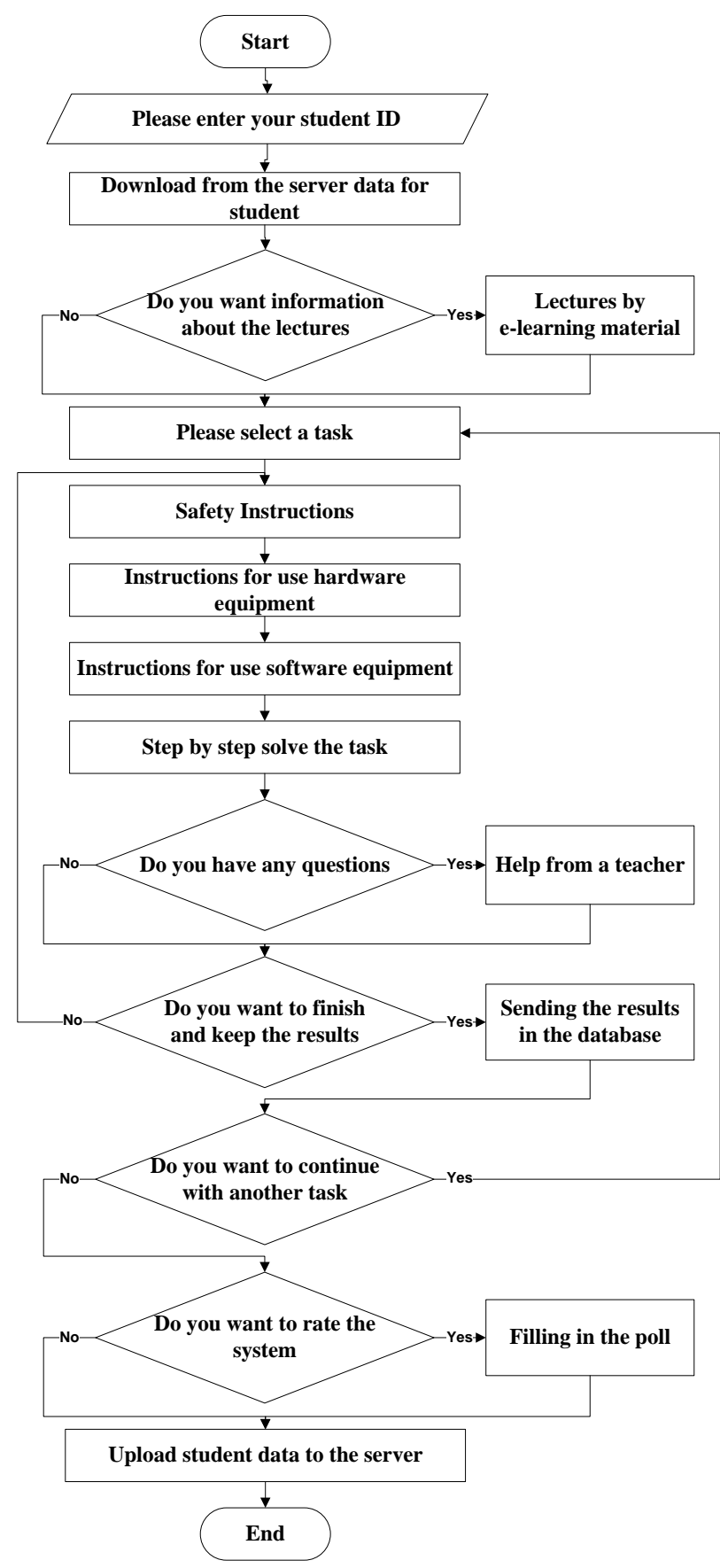

Fig. 3 Flowchart of the educational system

Presented algorithm is an example of the working of VPA as this model can be supplemented with additional functionalities. The main idea is VPA includes within itself software required for the main unit and software for individual sensors and modules, but to present all of this with interactive friendly interface. Solving problems step by step allows students to demonstrate their knowledge in practice and thus gain experience of solving problems in the real process. The teacher plays an important role in solving the tasks of this method as only intervene when a student needs help.

The exercises should be prepared by the teaching team in a given discipline and integration of new hardware modules and sensors will complement and software library for working with them. Each exercise should be developed accordingly method "step by step" and contain relevant instructions for each stage can be applied in the model.

\section{INTEGRATION WITH APPLICATION PLATFORMS}

In order to deal with the big data collected from VPA, the equipment also has compatibility with platforms such as LabVIEW and SAP HANA for additional system capabilities. SAP HANA is specialized for collect all your data needs, removing the burden of maintaining separated legacy systems and soiled data, so one may run simple in this new digital economy [8]. This system can be used for storing and analyzing data from sensors and modules for different tasks. Then from the system can generate application where it is displayed in the appropriate element type of stored data. As shown in Fig. 4, we use the educational device to monitor a dynamic balancing machine.

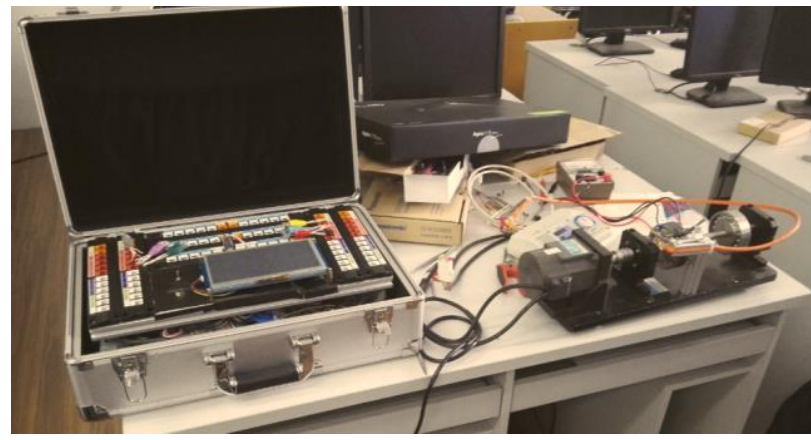

Fig. 4 Dynamic balancing machine

The educational device collects temperature, rotating speed, and vibration signals in three axes under both normal and abnormal working condition. In the abnormal working condition, types of fault such as damage on bearing, wear in linker and misalignment between the center of the spindle and platform will be injected. Fig. 5 shows the data collected from the educational device.

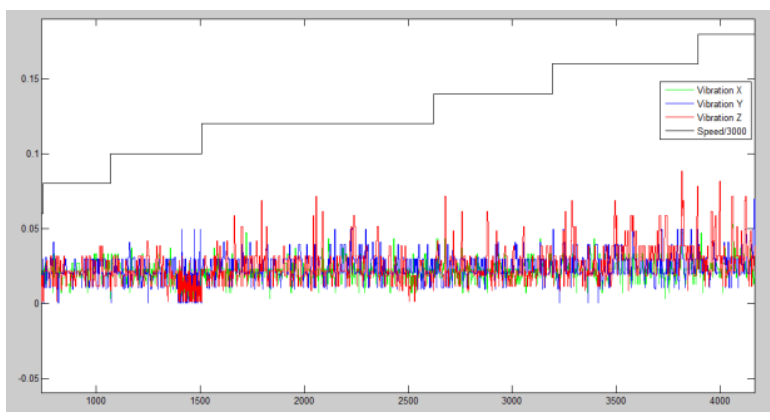

Fig. 5 Data collected from the educational device 


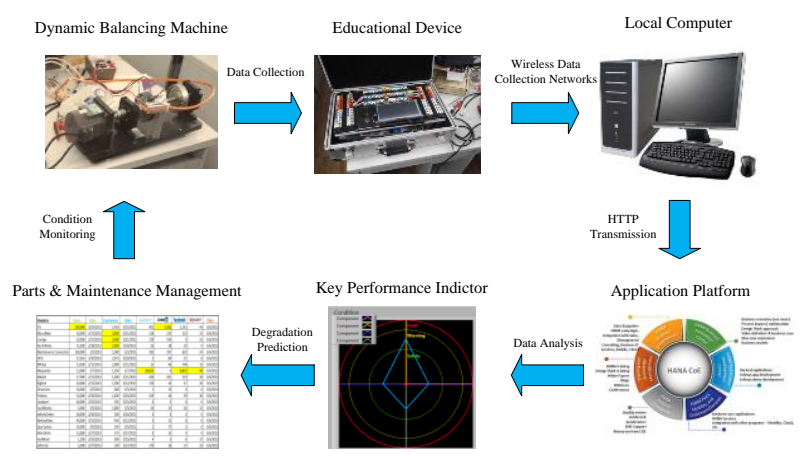

Fig. 6 Structure of integration with application platforms

As shown in Fig. 6, after the educational device collect the data from dynamic balancing machine, the data will be transported to application platform through HTTP Transmission. Afterwards, some data analysis will be conducted to extract the feature and form the Key Performance Indictor (KPI) for Degradation Prediction. So the working condition of the machine can be evaluated and maintenance can be scheduled in advance.

\section{CONCLUSIONS AND FUTURE WORK}

The creation of hardware equipment with built-in virtual personal with the above options will largely solve the problem of integrating the latest hardware platforms in training in the educational system. This model can be realized with almost all commercially available platforms for developers and can be used by professionals to solve everyday task. Where they were received very good feedback as to the operation of the module with interactive interface and the connection with application platform.

The next stage is to develop tasks for many disciplines and testing them with students and analyzing the resulting data. It will also analyze how this model affects the training of new digital generation.

\section{REFERENCES}

[1] https://www.raspberrypi.org/picademy/

[2] http://www.bananapi.org/

[3] http://beagleboard.org/bone

[4] Arduino, https://blog.arduino.cc/2016/05/20/bringing-technology-intothe-hands-of-teachers-and-students/

[5] Intel ${ }^{\circledR}$ Edison Compute Module, Boards, and Kits, http://www.intel.com/content/www/us/en/do-it-yourself/edison.html

[6] A. S. Smrikarov, T. I. Vassilev and Y. A.Stefanov, "Adapting the Education System to the Digital Generation", IN: Proceedings of the International Conference on e-Learning' 15, Berlin, Germany, , pp. 10-15, ISBN 2367-6698, 2015.

[7] Я. А. Стефанов, А.С. Смрикаров, “Една концепция за създаване на виртуален персонален асистент на студента". В: Научни трудове на Русенския университет, Русе, Издателски центьр на РУ, Russian, 2015.

[8] SAP - HANA, https://hana.sap.com/abouthana.html 